\title{
Risk of Nephrolithiasis and Nephrocalcinosis in Patients with Chronic Hypoparathyroidism: A Retrospective Cohort Study
}

\author{
Markus Ketteler · Kristina Chen · Elvira O. Gosmanova • \\ James Signorovitch · Fan Mu · Joshua A. Young · Nicole Sherry • \\ Lars Rejnmark
}

Received: November 24, 2020 / Accepted: February 3, 2021 / Published online: March 11, 2021

(C) The Author(s) 2021

\section{ABSTRACT}

Introduction: Chronic hypoparathyroidism managed with conventional treatment, comprising oral administration of calcium and active vitamin $\mathrm{D}$, has been associated with renal complications, including nephrolithiasis and nephrocalcinosis. Further larger-scale studies are needed to examine these risks. This study

Supplementary Information The online version contains supplementary material available at https:// doi.org/10.1007/s12325-021-01649-2.

M. Ketteler $(\bowtie)$

Department of General Internal Medicine and

Nephrology, Robert-Bosch-Krankenhaus, Stuttgart, Germany

e-mail: Markus.Ketteler@rbk.de

M. Ketteler

University of Split School of Medicine (USSM), Split, Croatia

K. Chen · N. Sherry

Shire Human Genetic Therapies, Inc., A Takeda

Company, Lexington, MA, USA

E. O. Gosmanova

Division of Nephrology and Hypertension, Albany

Medical College, Albany, NY, USA

J. Signorovitch $\cdot$ F. Mu $\cdot$ J. A. Young

Analysis Group, Inc., Boston, MA, USA

L. Rejnmark

Aarhus University and Department of

Endocrinology and Internal Medicine, Aarhus

University Hospital, Aarhus, Denmark evaluated the risk of nephrolithiasis and nephrocalcinosis in patients with chronic hypoparathyroidism.

Methods: A retrospective cohort study using a managed care claims database in the United States from January 2007 to June 2017. Included patients were those with chronic hypoparathyroidism (excluding those receiving parathyroid hormone) and randomly selected patients without hypoparathyroidism over a maximum of 5-year follow-up. The main outcome measures were nephrolithiasis, identified by diagnosis codes or procedure codes for removing kidney stones, and nephrocalcinosis, identified by diagnosis codes.

Results: The nephrolithiasis analyses included 8097 adult patients with hypoparathyroidism and 40,485 adult patients without hypoparathyroidism. After excluding patients with a diagnosis of nephrocalcinosis at baseline, nephrocalcinosis analyses included 8051 patients with hypoparathyroidism and 40,466 patients without hypoparathyroidism. During 5 years of follow-up, patients with chronic hypoparathyroidism had significantly increased risk of nephrolithiasis and nephrocalcinosis in Kaplan-Meier analysis compared with patients without hypoparathyroidism (both $P<0.001$ ). In the adjusted analyses, chronic hypoparathyroidism was associated with higher risks of nephrolithiasis (hazard ratio [HR], 1.81; 95\% confidence interval [CI] 1.60-2.04) and nephrocalcinosis (HR, 6.94; 95\% CI 
4.41-10.92). A sensitivity analysis restricted to patients with at least one kidney imaging examination showed that $2.6 \%$ of patients $(n=59)$ with hypoparathyroidism and $0.5 \%$ of patients $(n=20)$ without hypoparathyroidism (ratio, $\quad 5.5 ; \quad P<0.001$ ) developed nephrocalcinosis.

Conclusions: This large retrospective cohort study showed a statistically significant and clinically meaningful increased risk of nephrolithiasis and nephrocalcinosis in patients who have chronic hypoparathyroidism compared with those who do not have chronic hypoparathyroidism.

Keywords: Active vitamin D; Calcium; Chronic hypoparathyroidism; Chronic kidney disease; Kidney stones; Nephrocalcinosis; Nephrolithiasis

\section{Key Summary Points}

Why carry out this study?

Chronic hypoparathyroidism is a rare endocrine disorder; few large-scale studies have evaluated the long-term effects of chronic hypoparathyroidism on renal function.

This retrospective cohort study examined risks of development of nephrolithiasis and nephrocalcinosis in patients with chronic hypoparathyroidism.

\section{What was learned from the study?}

Patients with chronic hypoparathyroidism had a significant and clinically meaningful increased risk of developing nephrolithiasis and nephrocalcinosis, compared with those without hypoparathyroidism.

Further studies are warranted to better understand the potential mechanisms for the relationship of chronic hypoparathyroidism and its management with the observed risk of these conditions.

\section{DIGITAL FEATURES}

This article is published with digital features, including summary slide, to facilitate understanding of the article. To view digital features for this article go to https://doi.org/10.6084/ m9.figshare.13686823.

\section{INTRODUCTION}

Hypoparathyroidism is a rare endocrine disorder caused by absolute or relative deficiency of parathyroid hormone (PTH) [1, 2]. Chronic hypoparathyroidism is estimated to affect approximately 60,000 individuals in the United States and is associated with a high symptom burden, impaired quality of life, and increased risk of various long-term complications, including renal disease, cardiovascular conditions, cataracts, infections, and neuropsychiatric disorders [3-7].

Parathyroid hormone is an essential regulator of calcium and phosphate homeostasis through its actions on bone, intestine, and kidney $[8,9]$. Calcium-sensing receptors in the parathyroid glands signal the release of PTH when serum calcium levels fall [9]. In turn, PTH mobilizes calcium release from skeletal stores, increases calcium absorption from the intestine via stimulation of calcitriol synthesis, and, in the kidney, increases calcium reabsorption and phosphate excretion [9]. When PTH levels are inappropriately low or absent, normal calcium and phosphate homeostasis cannot be maintained, resulting in hypocalcemia and hyperphosphatemia, the characteristic biochemical findings of hypoparathyroidism [8].

Conventional management of chronic hypoparathyroidism involves supplementation with oral administration of calcium and active vitamin $\mathrm{D}$, which are prescribed to control the serum calcium concentration [10, 11], but many patients remain symptomatic or do not reach treatment goals $[9,10]$. With chronic use of added calcium without the renal calciumsparing effect of $\mathrm{PTH}$, patients may become hypercalciuric $[9,10]$; they also are at increased risk of renal complications, which are the most 
common long-term complications of chronic hypoparathyroidism [12].

Few studies have examined the risk of longterm renal complications associated with chronic hypoparathyroidism [7, 12-14]. A retrospective cohort study found that patients with postsurgical hypoparathyroidism $(n=668)$ had an almost fivefold increased risk of nephrolithiasis and renal insufficiency compared with age- and gender-matched controls $(n=2064)$ [13]. The current retrospective cohort study evaluated the association between chronic hypoparathyroidism and the risk of nephrolithiasis and nephrocalcinosis using a large managed care claims database.

\section{METHODS}

\section{Data Source and Study Design}

Data for this retrospective study were extracted from a large United States-based managed care claims database covering commercial (employer database) and Medicare Advantage beneficiaries between January 2007 and June 2017, representing more than 14 million individuals per year. Claims are sourced from a single national health insurance carrier. Because this study used deidentified licensed data from a Health Insurance Portability and Accountability Act (HIPAA)-compliant managed care database, ethics committee approval, and informed consent were not required.

Patients with and those without chronic hypoparathyroidism from the managed care claims database were selected into separate cohorts. Inclusion in both cohorts required patients to be at least 18 years of age on the index date, had 6 months or more of continuous eligibility before the index date, and had no evidence of treatment with recombinant PTH. Patients with chronic hypoparathyroidism had two or more medical claims with diagnoses of hypoparathyroidism at least 6 months apart. The index date for those in the chronic hypoparathyroidism cohort was the date of the first diagnosis of hypoparathyroidism at least 6 months after the initial hypoparathyroidism diagnosis. Patients without chronic hypoparathyroidism never had a diagnosis of hypoparathyroidism and had at least one medical claim for any condition other than hypoparathyroidism that satisfied all other selection criteria; the index date for these patients was defined as the date of a randomly selected eligible claim.

The baseline period for both cohorts comprised the 6 months before the index date. Patients were followed up for a maximum of 5 years after the index date or until the end of continuous eligibility (i.e., uninterrupted coverage by the insurance provider), whichever came first; this defines the study period.

\section{Outcome Measures and Assessments}

Nephrolithiasis was identified by International Statistical Classification of Diseases and Related Health Problems, 9th (ICD-9) or 10th (ICD-10) Revision, Clinical Modification (CM) ICD-9-CM or ICD-10-CM diagnosis codes, or Procedure Coding System (PCS) ICD-9-PCS or ICD-10-PCS procedure codes; the Healthcare Common Procedure Coding System (HCPCS); and Current Procedural Terminology (CPT) codes (Supplementary Table 1). Presence of nephrocalcinosis in patients without a diagnosis of nephrocalcinosis during the baseline period was identified by ICD-9-CM or ICD-10-CM diagnosis codes (Supplementary Table 1).

Hypercalcemia, hypercalciuria, hyperphosphatemia, hypocalcemia, and other comorbid conditions were identified using ICD-9-CM and ICD-10-CM diagnosis codes. Generic product identifier codes were used to determine oral thiazide diuretic use, and instances of kidney imaging were identified using ICD-9 and ICD10 procedure codes or CPT codes.

\section{Statistical Analyses}

Descriptive statistics were used to describe demographic and clinical characteristics at baseline. Between-cohort comparisons of baseline characteristics were conducted using Wilcoxon rank sum tests (continuous variables) or chi-square tests (categorical variables). The Kaplan-Meier method was used to determine 
time to event for each clinical outcome, with time to event estimated from the index date to the date of the first instance of the clinical outcome. Patients without the clinical outcome were censored at the end of insurance eligibility or 5 years after the index date, whichever occurred first. Comparisons were made between cohorts using log-rank tests. Unadjusted and adjusted Cox proportional hazards models were used to determine hazard ratios (HRs). Based on clinical input during development of the analysis, adjusted models were controlled for baseline demographic characteristics (age, sex, race, region, index year) and outcome-relevant clinical characteristics.

For the analysis of nephrolithiasis, outcomerelevant baseline clinical characteristics for adjustment in multivariable analysis included comorbidities (gout, hypercalciuria, hypertension, nephrolithiasis, diabetes) and use of thiazide diuretics. In the primary analysis, all patients were assessed regardless of baseline nephrolithiasis. Additional exploratory analyses were performed (1) among patients without nephrolithiasis in the baseline period and (2) among patients without nephrolithiasis in the baseline period without adjusting for baseline oral thiazide diuretic use.

For the analysis of nephrocalcinosis, patients without a diagnosis of nephrocalcinosis during the baseline period were selected for the assessment. The adjusted Cox proportional hazards model for nephrocalcinosis controlled for hypercalciuria in addition to demographic characteristics at baseline.

A sensitivity analysis was conducted among all patients in each cohort who had undergone at least one kidney imaging procedure during the study. The proportions of patients in these subsets with a diagnosis of nephrocalcinosis during the study were calculated; dichotomous variables were compared between cohorts using chi-square tests.

\section{RESULTS}

Following sample selection based on relevant eligibility criteria, 8097 patients with chronic hypoparathyroidism and 40,485 patients without hypoparathyroidism were included in the nephrolithiasis analyses (Fig. 1); 8051 and 40,466 patients with and without chronic hypoparathyroidism, respectively, were included in the nephrocalcinosis analysis after excluding patients with baseline nephrocalcinosis.

Baseline characteristics are shown in Table 1. Patients who have chronic hypoparathyroidism compared with those who do not were older (58.6 versus 47.3 years) and a higher proportion were female $(76.2 \%$ versus $54.4 \%$; both comparisons, $P<0.001)$. Most patients were White (64.7\% versus $63.1 \%$ for those with and without chronic hypoparathyroidism, respectively). A higher percentage of the cohort with versus without chronic hypoparathyroidism had comorbidities, including hypertension, hypercalciuria, type 2 diabetes, heart failure, nephrolithiasis, gout, type 1 diabetes, and nephrocalcinosis (all comparisons, $P<0.001$ ). Thiazide diuretics were used by $17.1 \%$ of patients in the cohort with chronic hypoparathyroidism compared with $9.9 \%$ of those without chronic hypoparathyroidism $(P<0.001)$.

Patients with chronic hypoparathyroidism had a significantly increased risk of nephrolithiasis compared with patients without chronic hypoparathyroidism in the Kaplan-Meier analysis $(P<0.001$; Fig. $2 \mathrm{a})$. When the analysis was performed among patients without nephrolithiasis during the baseline period, the percentage of patients with nephrolithiasis was reduced, but the risk remained significant $(P<0.001$; Fig. 2b). A diagnosis of chronic hypoparathyroidism was significantly associated with increased likelihood of nephrolithiasis in both unadjusted (HR, 2.44 [95\% confidence interval: CI 2.20-2.71]; $P<0.001$ ) and adjusted (HR, 1.81 [95\% CI 1.60-2.04]; $P<0.001$ ) Cox models (Table 2). In sensitivity analyses (1) among patients without nephrolithiasis in the baseline period and (2) among patients without nephrolithiasis in the baseline period and without adjusting for baseline oral thiazide diuretic use, the risk of developing nephrolithiasis remained significant and increased slightly (both $P<0.001$; Table 2 ) 
(a) Patients With Hypoparathyroidism

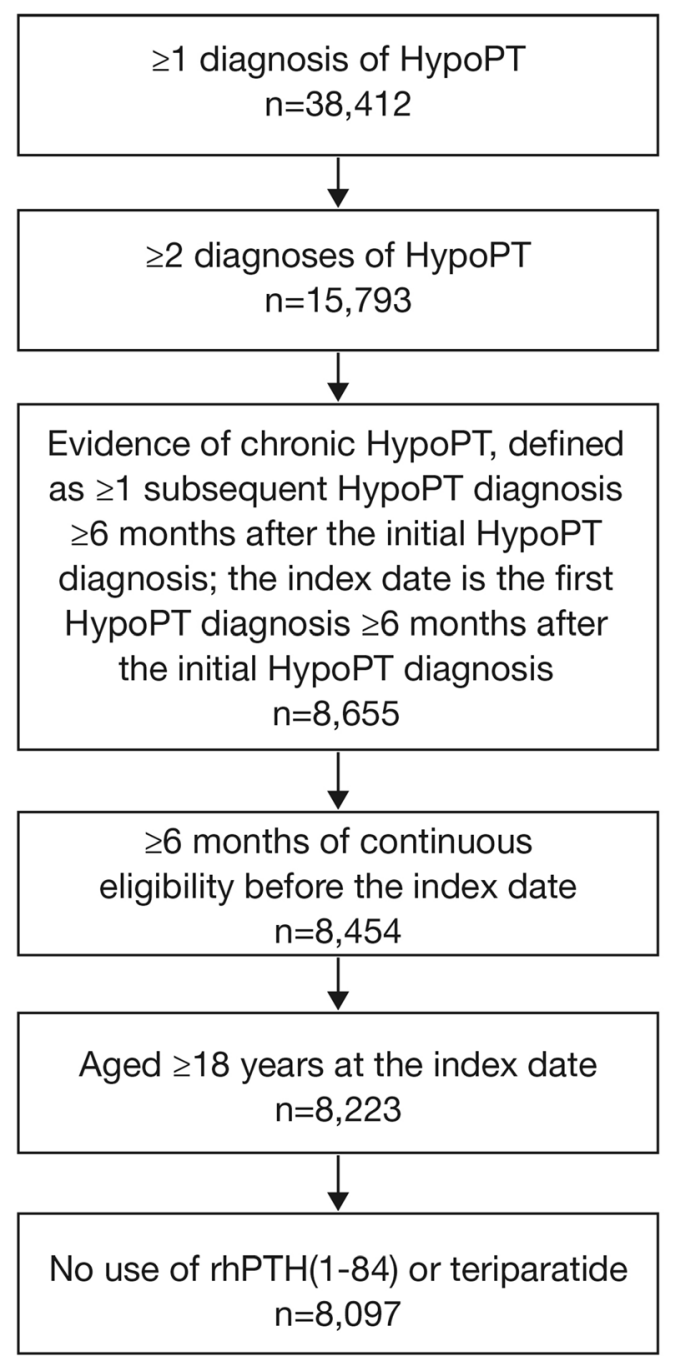

(b) Patients Without Hypoparathyroidism

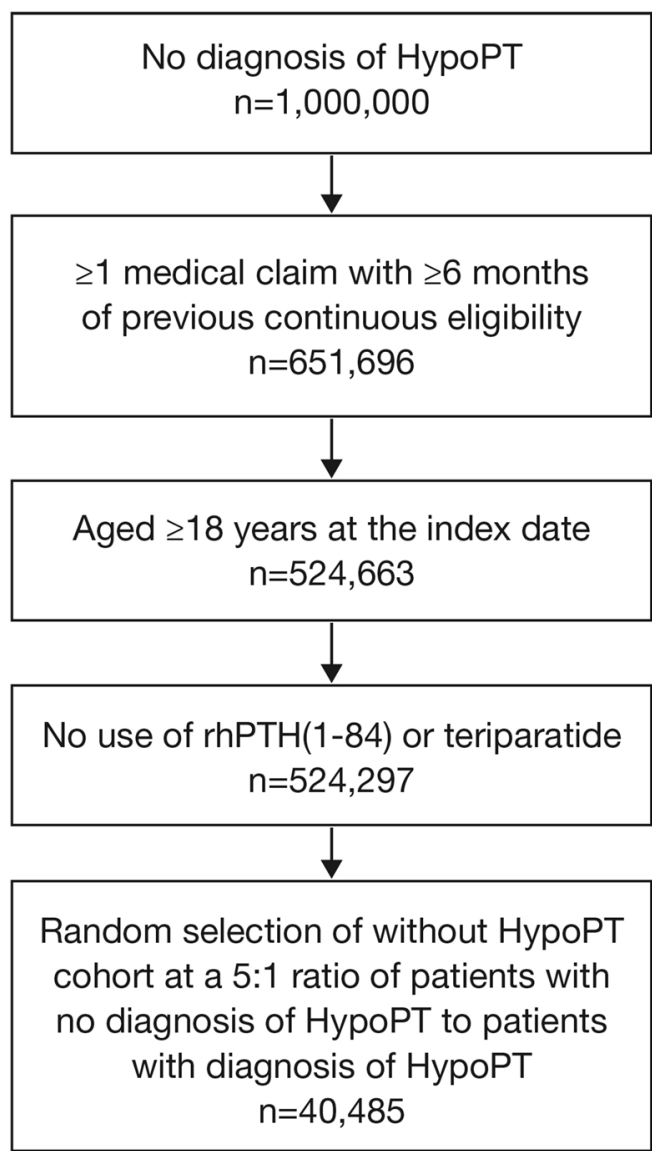

Fig. 1 Sample selection of patients $\mathbf{a}$ with and $\mathbf{b}$ without chronic hypoparathyroidism. HypoPT hypoparathyroidism, rhPTH(1-84) recombinant human parathyroid hormone (1-84)

for patients with chronic hypoparathyroidism compared with those without.

Kaplan-Meier analysis showed that the risk of nephrocalcinosis was significantly higher in patients who have chronic hypoparathyroidism compared with those who do not $(P<0.001$; Fig. 3). Chronic hypoparathyroidism was significantly associated with increased HR of nephrocalcinosis in both unadjusted (HR, 10.37 [95\% CI 6.96-15.45]; $P<0.001)$ and adjusted (HR, $6.94 \quad[95 \%$ CI $4.41-10.92] ; \quad P<0.001)$ models (Table 2).
The sensitivity analysis included 2277 patients $(28.1 \%)$ with chronic hypoparathyroidism and 4213 patients (10.4\%) without chronic hypoparathyroidism and who had undergone at least one kidney imaging procedure for any nonspecified reason during the study (total number of kidney imaging procedures was 5148 and 7211, respectively). Within this subset, 59 patients $(2.6 \%)$ with chronic hypoparathyroidism and 20 patients (0.5\%) without chronic hypoparathyroidism were 
Table 1 Baseline characteristics among patients with or without chronic hypoparathyroidism

\begin{tabular}{|c|c|c|c|}
\hline Characteristic & $\begin{array}{l}\text { With } \\
\text { hypoparathyroidism } \\
n=8097\end{array}$ & $\begin{array}{l}\text { Without } \\
\text { hypoparathyroidism } \\
n=40,485\end{array}$ & $P$ value \\
\hline Age at index date, mean (SD), years & $58.6(16.3)$ & $47.3(18.0)$ & $<0.001$ \\
\hline Female sex, $n(\%)$ & $6173(76.2)$ & $22,043(54.4)$ & $<0.001$ \\
\hline \multicolumn{4}{|l|}{ Race, $n(\%)$} \\
\hline White & $5242(64.7)$ & $25,560(63.1)$ & 0.006 \\
\hline Hispanic/Latino & $833(10.3)$ & $3951(9.8)$ & 0.145 \\
\hline Black & $711(8.8)$ & $3458(8.5)$ & 0.482 \\
\hline Asian/Pacific Islander & $239(3.0)$ & $1555(3.8)$ & $<0.001$ \\
\hline Unknown & $1072(13.2)$ & $5961(14.7)$ & $<0.001$ \\
\hline \multicolumn{4}{|l|}{ Comorbidities, $n$ (\%) } \\
\hline Hypertension & $3535(43.7)$ & $10,216(25.2)$ & $<0.001$ \\
\hline Hypercalciuria & $1924(23.8)$ & $189(0.5)$ & $<0.001$ \\
\hline Type 2 diabetes & $1670(20.6)$ & $4378(10.8)$ & $<0.001$ \\
\hline Heart failure & $481(5.9)$ & $967(2.4)$ & $<0.001$ \\
\hline Nephrolithiasis & $268(3.3)$ & $535(1.3)$ & $<0.001$ \\
\hline Gout & $244(3.0)$ & $499(1.2)$ & $<0.001$ \\
\hline Type 1 diabetes & $230(2.8)$ & $453(1.1)$ & $<0.001$ \\
\hline Nephrocalcinosis & $46(0.6)$ & $19(<0.1)$ & $<0.001$ \\
\hline Use of oral thiazide diuretics, $n(\%)$ & $1388(17.1)$ & $4017(9.9)$ & $<0.001$ \\
\hline Use of NSAIDs, PPIs, and cimetidine, $n$ (\%) & $2097(25.9)$ & $6949(17.2)$ & $<0.001$ \\
\hline $\begin{array}{l}\text { Use of } \geq 1 \text { drug from the following classes: ACE inhibitors, } \\
\text { ARBs, and diuretics, } n(\%)\end{array}$ & $1902(23.5)$ & $5568(13.8)$ & $<0.001$ \\
\hline
\end{tabular}

$A C E$ angiotensin-converting enzyme, $A R B$ angiotensin II receptor blocker, NSAID nonsteroidal anti-inflammatory drug, $P P I$ proton pump inhibitor, $S D$ standard deviation

diagnosed with nephrocalcinosis (ratio, 5.5; $P<0.001)$.

\section{DISCUSSION}

Compared with patients who did not have hypoparathyroidism, patients with the condition were at greater risk of nephrolithiasis and nephrocalcinosis during the 5-year follow-up after adjusting for potential confounders. This study was larger than previous research examining hypotheses similar to ours that used smaller cohorts in specific settings $[7,12,13,15]$. A strength of the current study is that the data were drawn from a large, nationally representative cohort of patients with hypoparathyroidism, confirming and expanding on prior associations reported between hypoparathyroidism and nephrolithiasis and nephrocalcinosis. For instance, a Danish retrospective study with a follow-up time of 8 years 


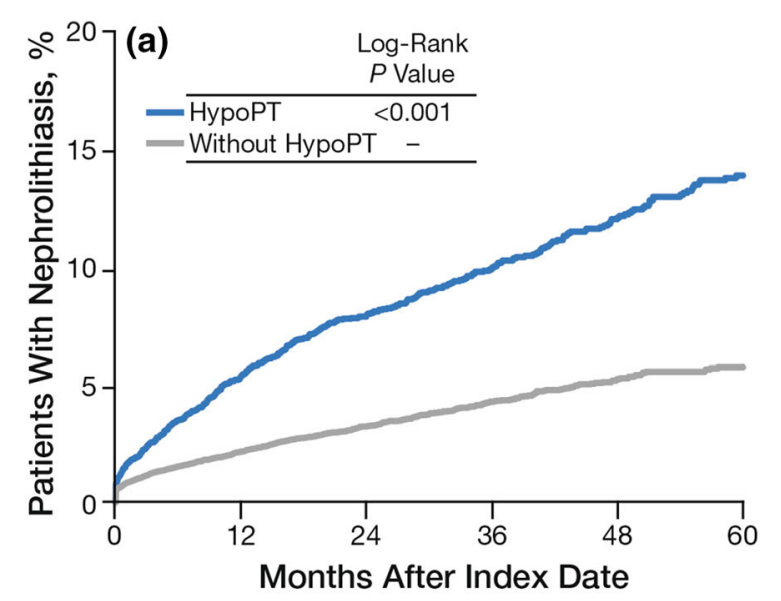

Number of patients with nephrolithiasis
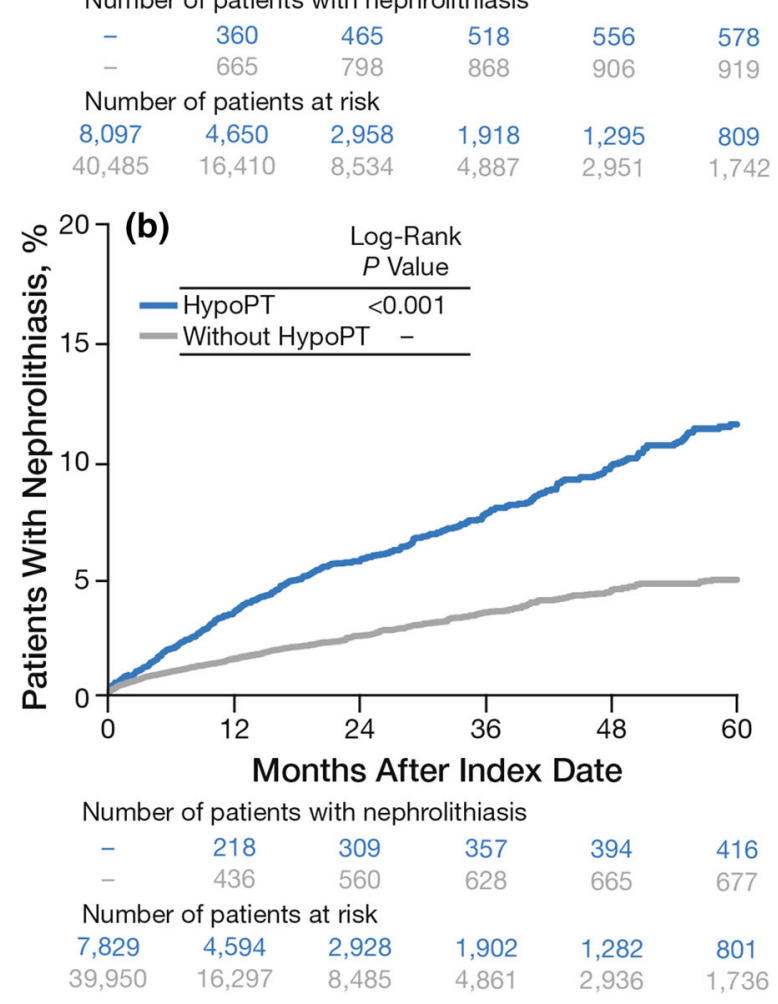

Fig. 2 Time to first instance ${ }^{a}$ of nephrolithiasis by patient cohort during the study period a among all patients and b among patients without nephrolithiasis during the baseline period. HypoPT hypoparathyroidism. ${ }^{\text {a Defined as }}$ the first diagnosis (by diagnosis or procedure codes) on or after the index date. Patients without a diagnosis during the study period were censored at the end of eligibility or 5 years after the index date, whichever occurred first

found that a cohort of 688 patients with postsurgical hypoparathyroidism had a nearly fivefold increased risk of nephrolithiasis compared with age- and gender-matched controls, which is comparable to the current findings [13]. Similarly, in a study comparing 90 patients with chronic postsurgical hypoparathyroidism treated with conventional therapy to 142 age- and sex-matched controls without hypoparathyroidism who were followed up for 7 years after surgery, those with hypoparathyroidism had an eightfold increase in the odds of having nephrolithiasis [16]. In a cross-sectional study of 25 women with postsurgical hypoparathyroidism treated with conventional therapy, approximately $8 \%$ had nephrolithiasis, whereas no age- and sex-matched patients in the control group had nephrolithiasis [17]. These findings are further supported by a long-term Massachusetts-based registry study of 120 patients with hypoparathyroidism treated with conventional therapy. In that study, nearly one-third of patients with hypoparathyroidism who underwent renal imaging were found to have either nephrolithiasis or nephrocalcinosis [12].

Although the current large-scale cohort study confirms the increased risk of nephrolithiasis and nephrocalcinosis in patients with hypoparathyroidism, the reasons for the elevation in risk remain unknown. One hypothesis is that treatment with conventional therapy consisting of oral calcium supplements and active vitamin $\mathrm{D}$ analogues, such as calcitriol, contributes to the risk. This regimen is commonly used for patients with hypoparathyroidism to maintain serum calcium concentrations in the lower end of the normal range (i.e., $8.0-8.5 \mathrm{mg} / \mathrm{dL}$ ) [10, 18-20] by increasing intestinal calcium absorption [21]. However, conventional therapy does not affect the mechanisms for renal calcium reabsorption and urinary phosphate excretion [1, 20]. Thus, it is hypothesized that use of calcium supplements or active vitamin $\mathrm{D}$ in the absence of the physiologic effect of PTH increases the risk of developing calcium-related kidney complications, such as nephrolithiasis and nephrocalcinosis, potentially through hypercalciuria $[9,12]$. In support of this hypothesis, Underbjerg et al. showed that the number of episodes of hypercalcemia was associated with increased risk of renal disease [15]. 
Table 2 Risk of nephrolithiasis and nephrocalcinosis associated with chronic hypoparathyroidism

\begin{tabular}{|c|c|c|c|}
\hline Outcome & HR & 95\% CI & $P$ value \\
\hline \multicolumn{4}{|l|}{ Nephrolithiasis ${ }^{\mathrm{a}}$} \\
\hline Unadjusted & 2.44 & $2.20-2.71$ & $<0.001$ \\
\hline Adjusted $^{\mathrm{b}}$ & 1.81 & $1.60-2.04$ & $<0.001$ \\
\hline Unadjusted, ${ }^{\mathrm{c}}$ among patients without baseline nephrolithiasis & 2.27 & $2.01-2.57$ & $<0.001$ \\
\hline Adjusted, ${ }^{\mathrm{b}}$ among patients without baseline nephrolithiasis & 1.97 & $1.71-2.27$ & $<0.001$ \\
\hline $\begin{array}{l}\text { Unadjusted, }{ }^{\mathrm{d}} \text { among patients without baseline nephrolithiasis and without adjusting for } \\
\text { baseline use of oral thiazide diuretics }\end{array}$ & 2.27 & $2.01-2.57$ & $<0.001$ \\
\hline $\begin{array}{l}\text { Adjusted, among patients without baseline nephrolithiasis and without adjusting for } \\
\text { baseline use of oral thiazide diuretics }\end{array}$ & 1.97 & $1.71-2.28$ & $<0.001$ \\
\hline \multicolumn{4}{|l|}{ Nephrocalcinosis ${ }^{a}$} \\
\hline Unadjusted & 10.37 & $6.96-15.45$ & $<0.001$ \\
\hline Adjusted $^{\mathrm{b}}$ & 6.94 & $4.41-10.92$ & $<0.001$ \\
\hline \multicolumn{4}{|c|}{$\begin{array}{l}C I \text { confidence interval, } H R \text { hazard ratio } \\
\text { a Patients without hypoparathyroidism served as the reference group for all analyses } \\
\text { b Multivariable Cox models adjusted for demographic (age, sex, race, region, and index year) and clinical (for } \\
\text { nephrolithiasis: nephrolithiasis, gout, hypercalciuria, hypertension, type } 1 \text { diabetes, type } 2 \text { diabetes, thiazide diuretic use; for } \\
\text { nephrocalcinosis: hypercalciuria) characteristics at baseline } \\
\text { c Multivariable Cox model conducted among patients without baseline nephrolithiasis } \\
\text { d Multivariable Cox model conducted among patients without baseline nephrolithiasis and without adjusting for baseline } \\
\text { oral thiazide diuretic use }\end{array}$} \\
\hline
\end{tabular}

It is likely that the course of hypoparathyroidism itself may increase the risk of nephrolithiasis and nephrocalcinosis. For example, the absence of the stimulatory effect of PTH on the reabsorption of calcium in the renal tubules could increase the risk of developing nephrolithiasis and nephrocalcinosis among patients with hypoparathyroidism [20]. Because conventional therapy is the current standard of care for patients with chronic hypoparathyroidism, it is difficult to determine whether the risk of nephrolithiasis and nephrocalcinosis associated with conventional therapy can be separated from that associated with the course of the disease.

Nephrolithiasis and nephrocalcinosis have related pathophysiology but differ in physical expression and manifestation. Nephrolithiasis is characterized by solid (mostly calcium) stones that appear in the kidney, whereas nephrocalcinosis consists of excess deposits of calcium salts within the renal tubules, tubular epithelium, or interstitium [22, 23]. Nephrolithiasis manifests as an acute, painful, and often recurring condition that may require hospitalization and costly treatment [24]. Owing to the recurring nature of nephrolithiasis events, all patients in this study were included in the nephrolithiasis analysis to evaluate time to first nephrolithiasis event during the study period regardless of baseline events. To account for the increased risk of nephrolithiasis among patients with nephrolithiasis during the baseline period, we adjusted for baseline nephrolithiasis and conducted a sensitivity analysis among patients without nephrolithiasis at baseline. On the other hand, nephrocalcinosis is a chronic condition that develops slowly without symptoms. Diagnostic imaging for nephrocalcinosis is important in the early 


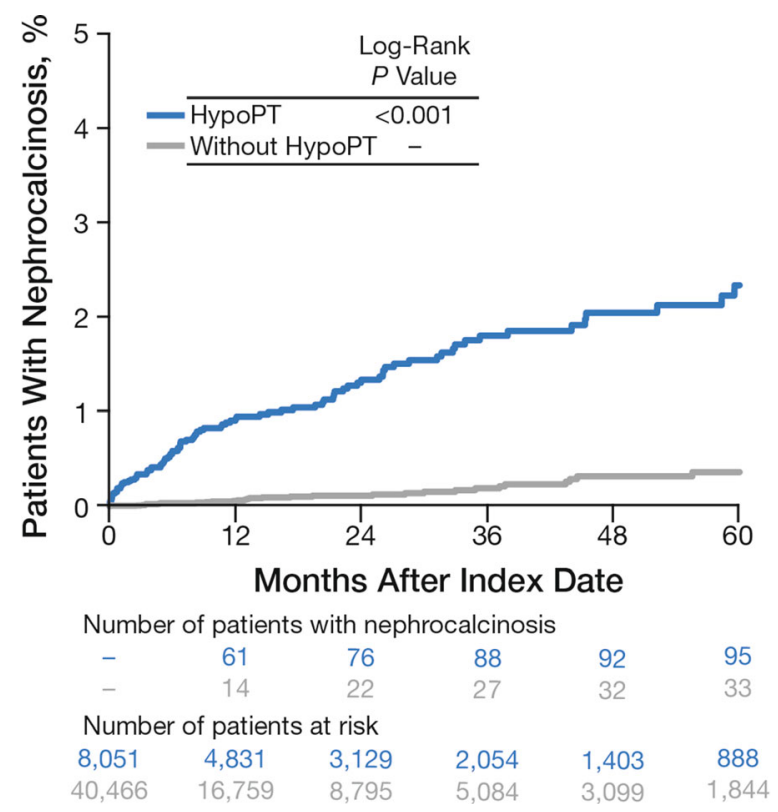

Fig. 3 Time to first instance ${ }^{a}$ of nephrocalcinosis by patient cohort during the study period among patients without nephrocalcinosis during the baseline period. HypoPT hypoparathyroidism. ${ }^{a}$ Defined as the first diagnosis (by diagnosis or procedure codes) on or after the index date in patients without diagnosis codes for nephrocalcinosis during the baseline period. Patients without a diagnosis during the study period were censored at the end of eligibility or 5 years after the index date, whichever occurred first

stages of the disease in patients with hypoparathyroidism who are at risk due to impairment of renal calcium reabsorption [23]. As a result of the chronic nature of this condition, patients with baseline nephrocalcinosis were excluded from the nephrocalcinosis analyses in order to examine new diagnoses of nephrocalcinosis during the study period.

For both the nephrolithiasis and nephrocalcinosis analyses, we adjusted for differences in baseline demographic characteristics (age, sex, race, region, and index year) and clinical profiles between the cohorts with and without hypoparathyroidism. Even after adjustment for potential confounders, hypoparathyroidism remained significantly associated with nephrolithiasis and nephrocalcinosis $(p<0.001)$. The statistically significant risk increase has clinical significance for patients and treating physicians. Nephrocalcinosis is a relatively rare occurrence in the healthy population, and the HR of occurrence in the hypoparathyroidism population was almost seven. In contrast, nephrolithiasis occurs relatively frequently in the healthy population, and yet an HR near two was observed for patients who have chronic hypoparathyroidism compared with those who do not have chronic hypoparathyroidism. Although age and sex appeared to have limited effect, adjustment for known baseline outcome-relevant clinical comorbidities such as hypertension, thiazide diuretic use $[9,25]$, hypercalciuria and type 2 diabetes [13], slightly attenuated the association of hypoparathyroidism and nephrolithiasis. Baseline nephrolithiasis was associated with a greatly increased risk of nephrolithiasis during the study period, which was expected owing to the frequency of nephrolithiasis recurrence. For the nephrocalcinosis outcome, baseline hypercalciuria attenuated the association with hypoparathyroidism but remained significant and was expected, as high renal calcium excretion is a potential mechanism causing nephrolithiasis and nephrocalcinosis [12].

Being asymptomatic, nephrocalcinosis is frequently underdiagnosed [23], which constrained our ability to capture all potential nephrocalcinosis events in the claims data. Consistent with findings that nephrocalcinosis is difficult to detect in the absence of renal imaging [12], we observed a very small number of nephrocalcinosis events in the cohorts of patients with and without hypoparathyroidism. Because of the small number of events, the resulting confidence intervals in our main analysis were wide, but results were statistically significant.

We conducted a sensitivity analysis among patients who received at least one kidney imaging procedure during the study period to address the possibility that patients with hypoparathyroidism may be more frequently monitored with renal imaging than patients without hypoparathyroidism and, therefore, may be more likely to receive nephrocalcinosis diagnoses. Among patients who received kidney imaging, there was a statistically significantly higher proportion of patients with hypoparathyroidism diagnosed with 
nephrocalcinosis compared with those without hypoparathyroidism. These findings strengthen the study conclusion that patients with hypoparathyroidism are at an increased risk of nephrocalcinosis.

Several important limitations should be considered when evaluating our study findings. First, there may be unobserved or unmeasured differences between the cohorts with and without hypoparathyroidism that were not accounted for in the analyses because of the observational study design. Nephrocalcinosis diagnoses may be underreported because nephrocalcinosis can be asymptomatic and often requires frequent monitoring to be detected [23]. Future studies in which patients are routinely monitored with high-resolution renal imaging are needed to comprehensively capture nephrocalcinosis events. Additionally, as with all studies using administrative claims databases, there may be miscoded or missing diagnoses, procedure data, and drug codes. There was a lack of access to matched imaging for these ICD-identified cases. The reasons why patients with chronic hypoparathyroidism had more kidney imaging procedures than those without hypoparathyroidism is unclear. It is possible that imaging studies in patients with hypoparathyroidism could be for both symptomatic disease and proactive screening for nephrolithiasis and nephrocalcinosis because of accumulating evidence of kidney complications in these patients. Conversely, in patients without hypoparathyroidism symptoms may have been the main driver for conducting imaging studies. However, the 1.3-fold increase in imaging procedures in patients with chronic hypoparathyroidism does not account for the 5.5-fold increase in a diagnosis of nephrocalcinosis among these patients in our sensitivity analysis. This study was conducted in the United States, but the resulting conclusions are unlikely to vary across different countries or regions; however, the magnitude of the results may differ owing to different distributions of effect modifiers across geographic areas. Lastly, because patient-level laboratory data were only available for a small subset of patients in the analysis population it was not possible to assess potential biomarkers or treatments that may mediate the relationship between hypoparathyroidism and nephrolithiasis and nephrocalcinosis. Thus, the mechanism of the observed findings merits further research using other data sources.

Our study has many strengths and improves on the research design of prior analyses that relied on smaller populations. Using a large, nationally representative insurance claims database, this study assessed much larger cohorts than most previous studies of hypoparathyroidism [13, 16, 17]: results from more than 8000 patients with hypoparathyroidism and more than 40,000 patients without it will likely be generalizable to patients with hypoparathyroidism in the United States. The study design assessing renal outcomes of related pathophysiology that differentially manifest provides evidence for the potential contributions of hypoparathyroidism to renal outcomes with costly and severe health consequences. Finally, the sensitivity analysis conducted for nephrocalcinosis in cohorts of patients with and without nephrocalcinosis at baseline with kidney imaging procedures performed during the study period showed an increased risk of nephrocalcinosis among patients with hypoparathyroidism.

\section{CONCLUSIONS}

This database analysis of a large retrospective cohort of patients in the United States showed that chronic hypoparathyroidism was associated with an increased risk of nephrolithiasis and nephrocalcinosis. Further research is warranted to better understand the potential mechanisms for the relationship of chronic hypoparathyroidism and its management with the observed risk of these conditions.

\section{ACKNOWLEDGEMENTS}

Funding. This research and the journal's Rapid Service and Open Access fees were funded by Shire Human Genetic Therapies, Inc., a Takeda company, Lexington, MA, USA. 
Authorship. All named authors meet the International Committee of Medical Journal Editors (ICMJE) criteria for authorship for this article, take responsibility for the integrity of the work as a whole, and have given their approval for this version to be published.

Medical Writing, Editorial, and Other Assistance. We thank Gary Curhan for his contributions to the study design and data analysis, and for comments during early drafts of the manuscript. Under the direction of the authors, editorial support was provided by Alan Storey, PhD, an employee of ICON plc (Marlow, Buckinghamshire, UK), and funded by Shire Human Genetic Therapies, Inc., a Takeda company, Lexington, MA, USA.

Prior Presentations. Initial data from this study were presented at the Endocrine Society Annual Meeting, March 23-26, 2019, New Orleans, LA, USA; and then subsequently at the 21st European Congress of Endocrinology, May 18-21, 2019, Lyon, France; and the Congress of Nephrology, October 10-13, 2019, Dusseldorf, Germany.

Disclosures. Markus Ketteler has served as a research investigator and consultant for Shire, a Takeda company. Kristina Chen is a former employee of Shire Human Genetic Therapies, Inc., a Takeda company, Lexington, MA, USA; current affiliation is Arena Pharmaceuticals, Boston, MA, USA. Elvira O. Gosmanova has served as a consultant for Shire, a Takeda company. Nicole Sherry is an employee of Shire Human Genetic Therapies, Inc., a Takeda company, Lexington, MA, USA. James Signorovitch, Fan $\mathrm{Mu}$ and Joshua A. Young are employees of Analysis Group, Inc., contracted by Shire Human Genetic Therapies, Inc., a Takeda company, to conduct this research. Lars Rejnmark has served as a consultant and speaker for Shire, a Takeda company.

Compliance with Ethics Guidelines. Because this study used deidentified licensed data from a HIPAA-compliant managed care claims database, ethics committee approval, and informed consent were not required.
Data Availability. The datasets generated during and/or analyzed during the current study are available from the corresponding author on reasonable request.

Open Access. This article is licensed under a Creative Commons Attribution-NonCommercial 4.0 International License, which permits any non-commercial use, sharing, adaptation, distribution and reproduction in any medium or format, as long as you give appropriate credit to the original author(s) and the source, provide a link to the Creative Commons licence, and indicate if changes were made. The images or other third party material in this article are included in the article's Creative Commons licence, unless indicated otherwise in a credit line to the material. If material is not included in the article's Creative Commons licence and your intended use is not permitted by statutory regulation or exceeds the permitted use, you will need to obtain permission directly from the copyright holder. To view a copy of this licence, visit http://creativecommons.org/licenses/by$\mathrm{nc} / 4.0 /$.

\section{REFERENCES}

1. Khan AA, Koch C, Van Uum SHM, et al. Standards of care for hypoparathyroidism in adults: a Canadian and international consensus. Eur J Endocrinol. 2019;180:P1-22.

2. Babey M, Brandi ML, Shoback D. Conventional treatment of hypoparathyroidism. Endocrinol Metab Clin N Am. 2018;47:889-900.

3. Powers J, Joy K, Ruscio A, Lagast H. Prevalence and incidence of hypoparathyroidism in the United States using a large claims database. J Bone Miner Res. 2013;28:2570-6.

4. Hadker N, Egan J, Sanders J, Lagast H, Clarke BL. Understanding the burden of illness associated with hypoparathyroidism reported among patients in the PARADOX study. Endocr Pract. 2014;20:671-9.

5. Sikjaer T, Rolighed L, Hess A, Fuglsang-Frederiksen A, Mosekilde L, Rejnmark L. Effects of PTH(1-84) therapy on muscle function and quality of life in hypoparathyroidism: results from a randomized controlled trial. Osteoporos Int. 2014;25:1717-26. 
6. Astor MC, Lovas K, Debowska A, et al. Epidemiology and health-related quality of life in hypoparathyroidism in Norway. J Clin Endocrinol Metab. 2016;101:3045-53.

7. Underbjerg L, Sikjaer T, Mosekilde L, Rejnmark L. The epidemiology of nonsurgical hypoparathyroidism in Denmark: a nationwide case finding study. J Bone Miner Res. 2015;30:1738-44.

8. Shoback DM, Bilezikian JP, Costa AG, et al. Presentation of hypoparathyroidism: etiologies and clinical features. J Clin Endocrinol Metab. 2016;101:2300-12.

9. Abate EG, Clarke BL. Review of hypoparathyroidism. Front Endocrinol (Lausanne). 2017;7:172.

10. Brandi ML, Bilezikian JP, Shoback D, et al. Management of hypoparathyroidism: summary statement and guidelines. J Clin Endocrinol Metab. 2016;101:2273-83.

11. Bilezikian JP, Khan A, Potts JT Jr, et al. Hypoparathyroidism in the adult: epidemiology, diagnosis, pathophysiology, target-organ involvement, treatment, and challenges for future research. J Bone Miner Res. 2011;26:2317-37.

12. Mitchell DM, Regan S, Cooley MR, et al. Long-term follow-up of patients with hypoparathyroidism. J Clin Endocrinol Metab. 2012;97:4507-14.

13. Underbjerg L, Sikjaer T, Mosekilde L, Rejnmark L. Cardiovascular and renal complications to postsurgical hypoparathyroidism: a Danish nationwide controlled historic follow-up study. J Bone Miner Res. 2013;28:2277-85.

14. Underbjerg L, Sikjaer T, Rejnmark L. Health-related quality of life in patients with nonsurgical hypoparathyroidism and pseudohypoparathyroidism. Clin Endocrinol (Oxf). 2018;88:838-47.

15. Underbjerg L, Sikjaer T, Rejnmark L. Long-term complications in patients with hypoparathyroidism evaluated by biochemical findings: a case-control study. J Bone Miner Res. 2018;33:822-31.
16. Meola A, Vignali E, Matrone A, Cetani F, Marcocci C. Efficacy and safety of long-term management of patients with chronic post-surgical hypoparathyroidism. J Endocrinol Invest. 2018;41:1221-6.

17. Arlt W, Fremerey C, Callies F, et al. Well-being, mood and calcium homeostasis in patients with hypoparathyroidism receiving standard treatment with calcium and vitamin D. Eur J Endocrinol. 2002;146:215-22.

18. Bilezikian JP, Brandi ML, Cusano NE, et al. Management of hypoparathyroidism: present and future. J Clin Endocrinol Metab. 2016;101:2313-24.

19. Bollerslev J, Rejnmark L, Marcocci C, et al. European Society of Endocrinology clinical guideline: treatment of chronic hypoparathyroidism in adults. Eur J Endocrinol. 2015;173:G1-120.

20. Shoback D. Hypoparathyroidism. N Engl J Med. 2008;359:391-403.

21. Khan MI, Waguespack SG, Hu MI. Medical management of postsurgical hypoparathyroidism. Endocr Pract. 2011;17:18-25.

22. Weigert A, Hoppe B. Nephrolithiasis and nephrocalcinosis in childhood-risk factor-related current and future treatment options. Front Pediatr. 2018;6: 98.

23. Boyce AM, Shawker TH, Hill SC, et al. Ultrasound is superior to computed tomography for assessment of medullary nephrocalcinosis in hypoparathyroidism. J Clin Endocrinol Metab. 2013;98:989-94.

24. Saigal CS, Joyce G, Timilsina AR, the Urologic Diseases in America Project. Direct and indirect costs of nephrolithiasis in an employed population: opportunity for disease management? Kidney Int. 2005;68:1808-14.

25. Taylor JM, Kieneker LM, de Borst MH, et al. Urinary calcium excretion and risk of chronic kidney disease in the general population. Kidney Int Rep. 2017;2:366-79. 